\title{
Waveguiding in two-dimensional piezoelectric phononic crystal plates
}

Cite as: J. Appl. Phys. 101, 114904 (2007); https://doi.org/10.1063/1.2740352

Submitted: 27 February 2007 . Accepted: 12 April 2007 . Published Online: 05 June 2007

J. O. Vasseur, A.-C. Hladky-Hennion, B. Djafari-Rouhani, F. Duval, B. Dubus, Y. Pennec, and P. A. Deymier

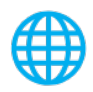

\section{ARTICLES YOU MAY BE INTERESTED IN}

Guiding and bending of acoustic waves in highly confined phononic crystal waveguides Applied Physics Letters 84, 4400 (2004); https://doi.org/10.1063/1.1757642

High- $Q$ micromechanical resonators in a two-dimensional phononic crystal slab Applied Physics Letters 94, 051906 (2009); https://doi.org/10.1063/1.3078284

Evidence of large high frequency complete phononic band gaps in silicon phononic crystal plates

Applied Physics Letters 92, 221905 (2008); https://doi.org/10.1063/1.2939097

\section{Challenge us.}

What are your needs for periodic signal detection?

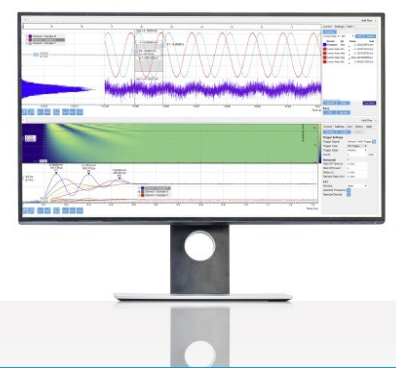

Zurich - Instruments 


\title{
Waveguiding in two-dimensional piezoelectric phononic crystal plates
}

\author{
J. O. Vasseur, ${ }^{\text {a) }}$ A.-C. Hladky-Hennion, B. Djafari-Rouhani, F. Duval, B. Dubus, and \\ Y. Pennec \\ Institut d'Electronique, de Micro-électronique et de Nanotechnologie, UMR CNRS 8520, Cité Scientifique, \\ 59652 Villeneuve d'Ascq Cedex, France \\ P. A. Deymier \\ Department of Materials Science and Engineering, University of Arizona, Tucson, Arizona 85721
}

(Received 27 February 2007; accepted 12 April 2007; published online 5 June 2007)

\begin{abstract}
We investigate the possibility of designing phononic crystal-based devices for telecommunication applications using materials commonly employed in microfabrication. We focus our attention on a phononic crystal made of a square array of cylindrical holes drilled in an active piezoelectric PZT5A matrix. Two different structures are considered, namely, a freestanding phononic crystal plate and a plate deposited on a silicon substrate. The geometrical characteristics of the phononic crystal plates (lattice parameter and thickness) were chosen to ensure the existence of an absolute band gap around $1.5 \mathrm{GHz}$; a common frequency in radio frequency telecommunications. Computations of the dispersion curves of these active structures were conducted with the help of the finite element method. We demonstrate the existence of absolute band gaps in the band structure of the phononic crystal plates and, then, the possibility of guided modes inside a linear defect created by removing one row of air holes in the phononic crystal. In the case of the supported phononic crystal plates, we show the existence of an absolute forbidden band in the plate modes when the thickness of the substrate significantly exceeds the plate thickness. We discuss the conditions to realize waveguiding through a linear defect inside the supported plate. The present work provides evidences that phononic crystal properties can be integrated with existing silicon based microdevice technology.
\end{abstract} (C) 2007 American Institute of Physics. [DOI: 10.1063/1.2740352]

\section{INTRODUCTION}

The propagation of elastic waves in periodic composite media, such as phononic crystals, has been the object of many researchers' interest in the past decade. ${ }^{1-3}$ Phononic crystals are created by a two-dimensional or threedimensional periodic arrangement of inclusions in a matrix. Phononic crystals may exhibit absolute band gaps where the propagation of elastic waves is forbidden in all directions. These band gaps arise under certain conditions of contrast in density and elastic properties, composition, geometry of the array of inclusions, and inclusion shape. When the periodicity of a phononic crystal is broken, it is possible to create highly localized defects within the acoustic bang gap, which are analogous to localized modes in photonic crystals and to localized impurity states in semiconductors. Extended defects such as rows of different inclusions in the phononic lattice have been shown to guide elastic waves within the crystal band gap. Defect modes can then lead to functionalities such as filtering and multiplexing. ${ }^{4-8}$

In addition to bulk phononic crystals, e.g., composite media of infinite extent along the three spatial directions, semi-infinite phononic crystals with a free surface have been shown to support surface acoustic waves (SAWs). The periodicity of the crystal in the plane of the surface may open gaps in the SAW branches. For instance, the existence of SAW localized at the free surface of a semi-infinite two-

\footnotetext{
a) Author to whom correspondence should be addressed; electronic mail: jerome.vasseur@univ-lille1.fr
}

dimensional phononic crystal has been proven theoretically. $^{9-12}$ For this geometry, the parallel inclusions are of cylindrical shape and the considered surface is perpendicular to their axis. Various arrays of inclusions, ${ }^{9,10}$ crystallographic symmetries of the component materials, ${ }^{11}$ and also the piezoelectricity of one of the constituents ${ }^{12}$ were taken into account. On the experimental side, Wu et al. ${ }^{13}$ observed high frequency SAW with a pair of interdigitated transducers placed on both sides of a thick silicon plate in which a square array of holes was drilled. Similar experiments were conducted by Benchabane et al. on a two-dimensional square lattice piezoelectric phononic crystal etched in lithium niobate. ${ }^{14}$ These studies suggest that integration of phononic crystals with radio frequency SAW devices may lead to a significant enhancement of the telecommunication device's functionality.

Slabs of phononic crystals also possess the characteristic properties of their bulk and semi-infinite counterparts. Gaps form in the symmetric Lamb mode band structure of twodimensional phononic crystal plates composed of triangular arrays of $\mathrm{W}$ cylinders in a Si background. ${ }^{15}$ Zhang et al. ${ }^{16}$ have shown the existence of gaps for acoustic waves propagating in an air/aluminium two-dimensional (2D) phononic crystal plate through laser ultrasonic measurements. Absolute band gaps appear in the band structure of phononic crystal plate composed of passive ${ }^{17,18}$ or piezoelectric materials. ${ }^{19}$ The existence of these absolute gaps depends strongly on the ratio of the plate thickness to the crystal periodicity with the largest gap occurring when this ratio is around 1. To obtain 


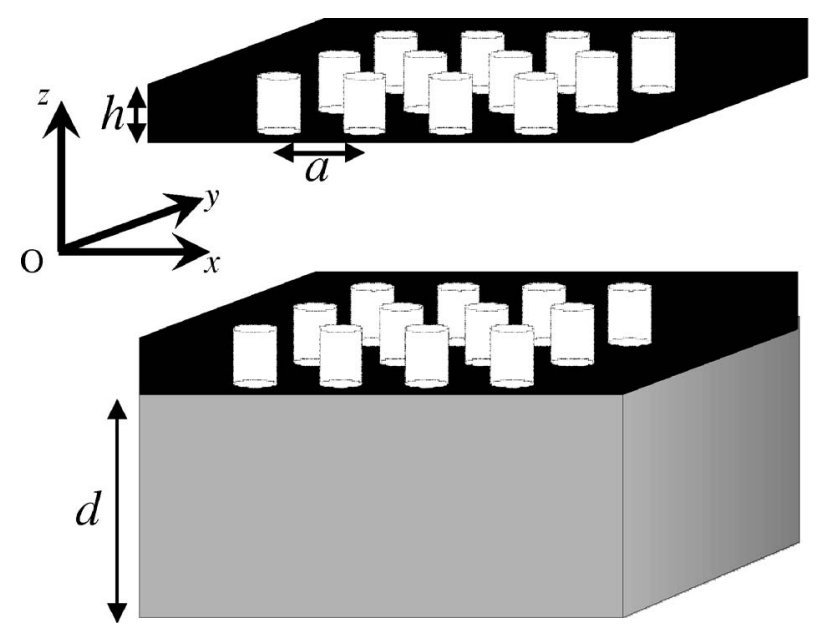

(a)

(b)

FIG. 1. (a) Freestanding phononic crystal plate of thickness $h$ and (b) phononic crystal plate of thickness $h$ deposited onto a homogeneous substrate of thickness $d$. The basic phononic crystal is composed of a square array of parallel cylindrical air inclusions (holes) of radius $R$ drilled in a piezoelectric matrix. The lattice parameter is $a$. The cylindrical air inclusions are assumed parallel to the $z$ axis of the Cartesian coordinates system $(O, x, y, z)$.

gaps at telecommunication frequencies of the order of gigahertz, the thickness of the plate should be of the order of micrometers. On the other hand, the realization of telecommunication devices requires, in general, the phononic crystal plates to be supported by a substrate.

In this paper, we report on the properties of piezoelectric phononic crystal freestanding plates and a plate supported on a silicon substrate. We focus on acoustic wave propagation in these structures and unravel the conditions under which absolute band gaps form for the plate modes. We also consider freestanding and supported phononic crystal plates containing rectilinear defects and we demonstrate the ability of the defect to guide acoustic waves with frequencies falling inside the forbidden band of the parent phononic crystal plate.

The paper is organized as follows. In Sec. II, we present the models of bulk phononic crystals, of phononic crystal plates, and of plates deposited on a substrate together with the finite element method used for calculating their band structures. In Sec. III, we discuss the behavior of the band structures with respect to the geometrical and physical parameters. Finally, some conclusions are drawn concerning the applicability of these structures to functional telecommunication devices.

\section{MODELS AND METHOD OF CALCULATION}

The basic phononic crystal used throughout this paper is composed of a square array of parallel cylindrical air inclusions (holes) of radius $R$ drilled in a PZT5A piezoelectric matrix. The cylindrical air inclusions are assumed parallel to the $z$ axis of the Cartesian coordinates system $(O, x, y, z)$. The filling factor $\beta$ of inclusions is defined as $\beta=\pi R^{2} / a^{2}$ and taken to be 0.7 to ensure the existence of an absolute band gap in the band structure of the bulk phononic crystal. ${ }^{17}$ We choose the lattice parameter $a$ of the periodic array equal to $0.77 \mu \mathrm{m}$ in order to locate the band gap around $1.5 \mathrm{GHz}$, a common frequency in telecommunications. The phononic
TABLE I. Physical characteristics of PZT5A: $\rho$ is the density, $\left(s^{E}\right)$ is the constant electric field elastic tensor, $(d)$ is the piezoelectric tensor, and $\left(\varepsilon^{S}\right)$ is the constant strain dielectric permittivity tensor. PZT5A is a hexagonal crystal, with $6 \mathrm{~mm}$ class symmetry.

\begin{tabular}{|c|c|c|}
\hline Constant & Unit & Value \\
\hline$\rho$ & $\mathrm{kg} / \mathrm{m}^{3}$ & 7750 \\
\hline$s_{11}^{E}$ & $\mathrm{~m}^{2} / \mathrm{N}$ & $1.640 \times 10^{-11}$ \\
\hline$s_{12}^{E}$ & & $-5.740 \times 10^{-12}$ \\
\hline$s_{13} E$ & & $-7.220 \times 10^{-12}$ \\
\hline$s_{33}^{E}$ & & $1.880 \times 10^{-11}$ \\
\hline$s_{44}^{E}$ & & $4.750 \times 10^{-11}$ \\
\hline$d_{31}$ & $\mathrm{C} / \mathrm{N}$ & $-1.710 \times 10^{-10}$ \\
\hline$d_{33}$ & & $3.740 \times 10^{-10}$ \\
\hline$d_{15}$ & & $5.840 \times 10^{-10}$ \\
\hline$\varepsilon_{11}^{S}$ & & $1036 \varepsilon_{0}$ \\
\hline$\varepsilon_{33}^{S}$ & & $939 \varepsilon_{0}$ \\
\hline
\end{tabular}

crystal plates are cut perpendicular to the $z$ axis. The thickness of the plate is denoted by $h$. Optimal conditions for gap formation in the plate modes are obtained for $h$ of the same order as $a{ }^{17}$ In the case of supported plates, the substrate is constituted by silicon, an ubiquitous material in microdevice manufacturing. In the numerical calculations, silicon was assumed of cubic crystallographic symmetry.

The thickness of the substrate is variable and defined as $d$ (see Fig. 1). The physical characteristics of PZT5A and Si are reported in Tables I and II.

The band structures were computed using the finite element method. ${ }^{20}$ According to the Bloch-Floquet relations, ${ }^{21}$ only the mesh of one unit cell is required. Dispersion curves of the bulk phononic crystal were calculated using a threedimensional (3D) unit cell: the structure is supposed to be infinite and uniform along the $z$ direction, infinite but periodic in the two other directions $x$ and $y$. This structure is named a 3D structure and, following the Bloch-Floquet theorem, a phase relation is applied on the six faces of the mesh. For the two other structures (plate, plate deposited onto a substrate), a 3D mesh is considered too but the structure is supposed to be of finite size along the thickness of the plate, periodic and infinite in the two other directions. Thus, it is named a 2D periodic structure and a phase relation is applied on only four faces of the mesh. The unit cell is meshed and divided into elements connected by nodes. Within this cell, a phase relation is applied on nodes separated by one period, defining boundary conditions between adjacent cells. The phase relation is related to the wave number of the incident wave in the periodic material. In this study, isoparametric elements are used, with 20 nodes for each element (hexahedron with one node at each corner and one node at the

TABLE II. Physical characteristics of silicon: $\rho$ is the density, $C_{11}, C_{12}$, and $C_{44}$ are the three independent elastic moduli of cubic silicon.

\begin{tabular}{lll}
\hline \hline Constant & Unit & \multicolumn{1}{c}{ Value } \\
\hline$\rho$ & $\mathrm{kg} / \mathrm{m}^{3}$ & 2331 \\
$C_{11}$ & $\mathrm{~N} / \mathrm{m}^{2}$ & $16.57 \times 10^{10}$ \\
$C_{12}$ & & $6.39 \times 10^{10}$ \\
$C_{44}$ & & $7.962 \times 10^{10}$ \\
\hline
\end{tabular}



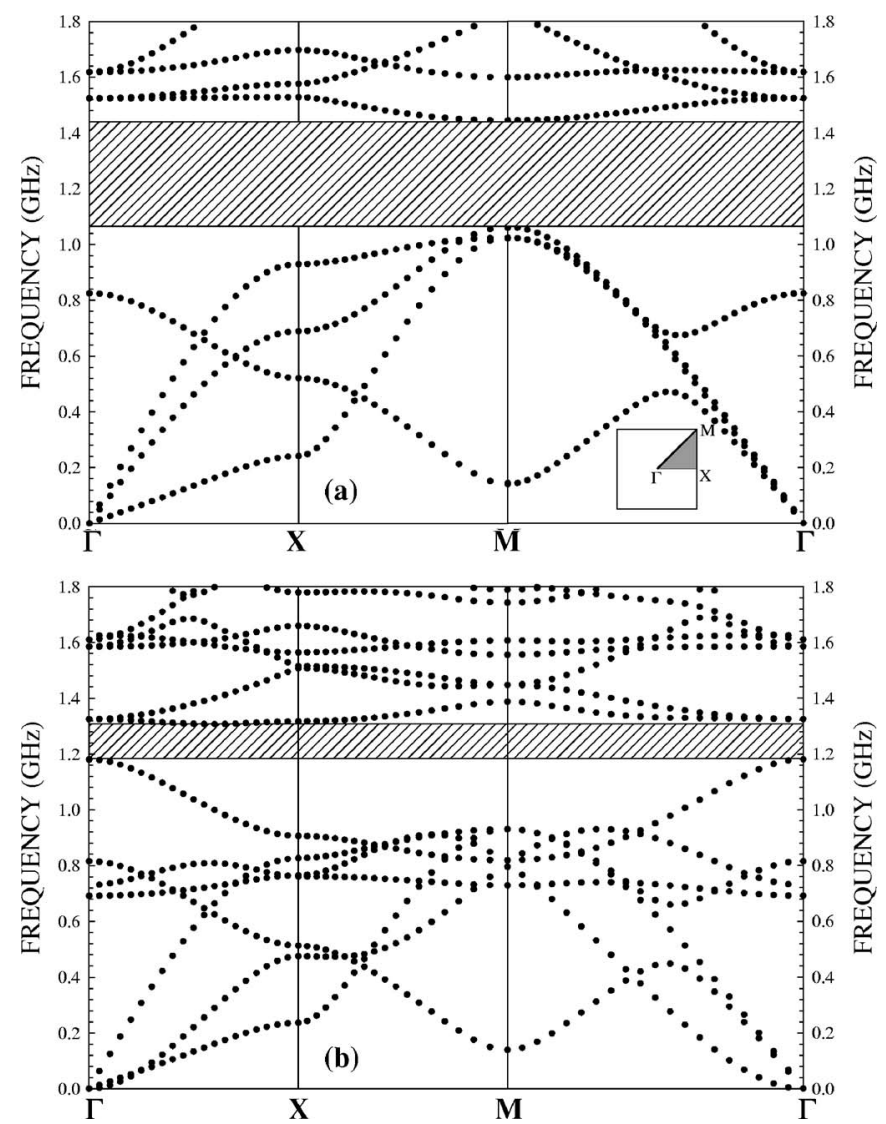

FIG. 2. Elastic band structures calculated with the finite element method for (a) the bulk 2D phononic crystal (structure of infinite extent along the three spatial directions) and (b) the freestanding phononic crystal plate of thickness $h=a=0.77 \mu \mathrm{m}$ made of a square array of holes drilled in a PZT 5A piezoelectric matrix with $\beta=0.7$. The inset represents the first Brillouin zone $(\Gamma X M)$ of the square array. The components of the wave vector at the $\Gamma, M$, and $M$ points are $(0,0),(\pi / a, 0)$, and $(\pi / a, \pi / a)$, respectively, with $\pi / a$ $=4.08 \mu \mathrm{m}^{-1}$.

middle of each edge), and a quadratic interpolation along element sides is considered, thanks to the three nodes per edge of the element. The band structures are rendered in terms of a frequency, function of the modulus of the wave vector, and are plotted along the principal directions of propagation of the 2D irreducible Brillouin zone (ГXM).

\section{RESULTS AND DISCUSSION}

\section{A. Freestanding PZT/air phononic crystal plate}

\section{Bulk phononic crystal and phononic crystal plate}

Figure 2(a) gives the band structure of the infinite phononic crystal, where only propagation perpendicular to the cylinders is taken into account. One can notice the existence of a band gap in the frequency range from 1.0596 to $1.4460 \mathrm{GHz}$. However, the band gap will be totally filled when taking into account the out-of-plane propagation. On the contrary, we have shown recently ${ }^{17}$ that a slab of a phononic crystal can display an absolute band gap provided its thickness $h$ is of the order of the in-plane lattice parameter $a$. This comes from the confinement of the modes in the slab that imposes limitations on the allowed values of the wave vector along the $z$ axis. Figure 2(b) gives the dispersion curves of such a slab when $h=a$; it shows an absolute
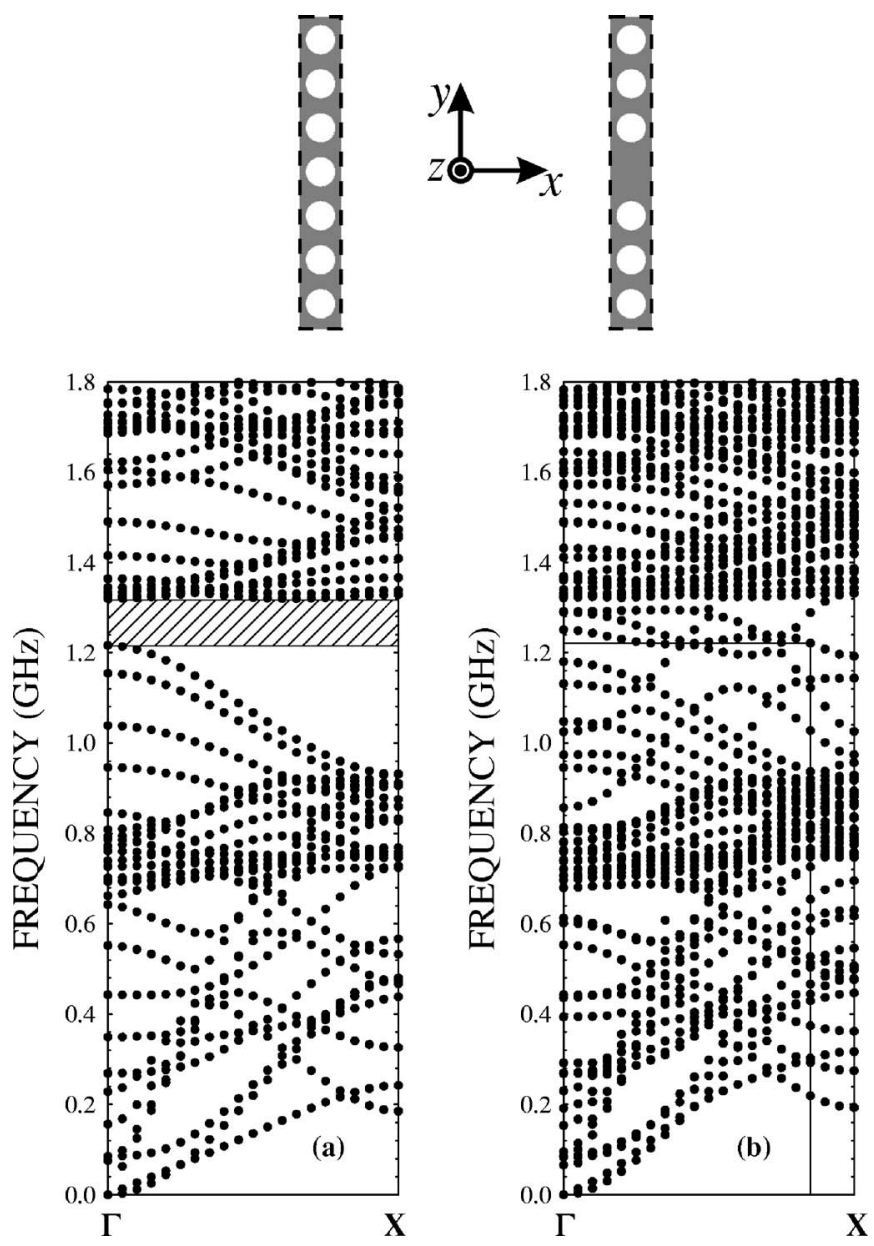

FIG. 3. Band structure along the $\Gamma X$ direction calculated with a supercell containing $1 \times 7$ unit cell, for (a) the perfect phononic crystal plate and (b) the phononic crystal plate containing a waveguide formed by filling the hole in the fourth unit cell. The inset depicts the $1 \times 7$ supercell considered in each case. The two straight lines indicate the location of the guided mode analyzed in Fig. 4.

band gap ranging from 1.1808 to $1.3072 \mathrm{GHz}$. The three lowest branches in the band structure, starting at the $\Gamma$ point, are quite similar to those of a homogeneous slab. They, respectively, correspond to the antisymmetric $A_{0}$ Lamb mode, the shear horizontal mode, and the symmetric $S_{0}$ Lamb mode.

\section{Waveguide in the freestanding plate}

We consider now the case of a rectilinear waveguide created inside the lead zirconate titanate (PZT)/air phononic crystal plate. A supercell containing seven unit cells in the $y$ direction is considered in the finite element calculations. The lattice parameter $a$ and the thickness of the plate $h$ are the same as for the perfect plate. Two systems are investigated. In the first system, the seven unit cells contain an air hole. In the second system, the hole in the fourth unit cell is filled with PZT5A, thus constituting a linear waveguide in the $x$ direction. Figures 3(a) and 3(b) show the band structure of the phononic crystal plate and the plate with a waveguide, respectively. We limit ourselves to the $\Gamma X$ path of the Brillouin zone that corresponds to the direction of propagation of the elastic waves inside the waveguide. Figure 3(a) exhibits 

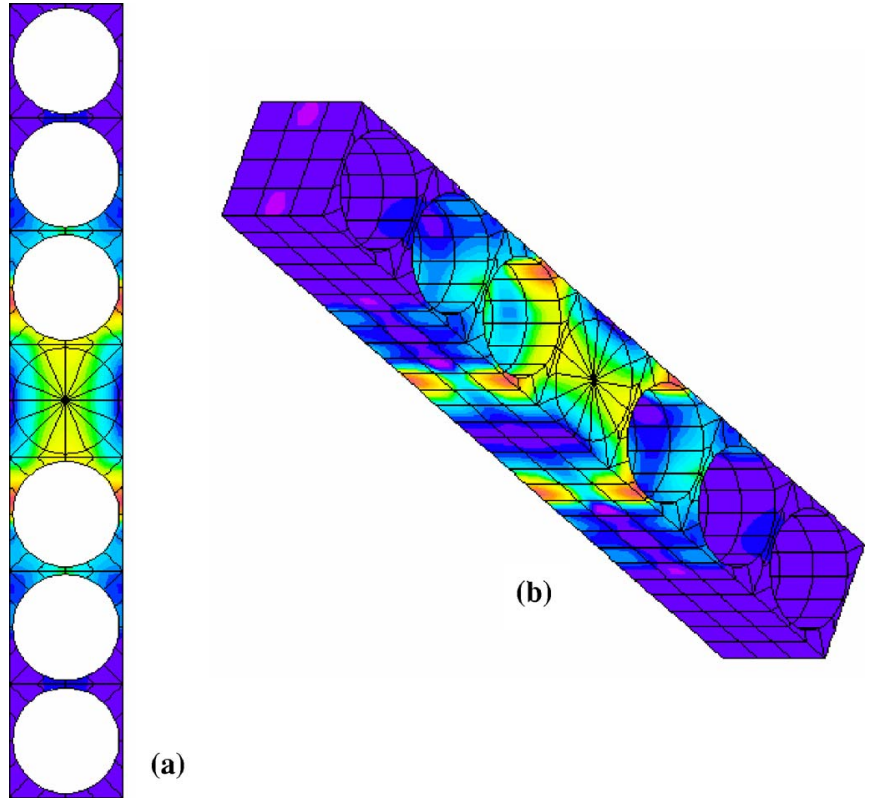

(a)

FIG. 4. (Color online) Maps of the modulus of the elastic displacement field for the waveguide mode with $f=1.221 \mathrm{GHz}$ and a wave vector of modulus $3.468 \mathrm{\mu m}^{-1}$; (a) top view and (b) three-quarter view. The red color corresponds to the maximum displacement, whereas the blue color corresponds to the minimum.

numerous additional branches with respect to those in Fig. 2(a) as the result of the folding of the bands in the $y$ direction due to the seven unit-cell periodicity in that direction. The band structure still shows the same forbidden band as the one displayed in Fig. 2(b). When a waveguide is introduced in the structure, guiding modes appear inside the band gap of the perfect phononic crystal plate, as illustrated in Fig. 3(b). More specifically, we illustrate in Fig. 4, the modulus of the displacement field for the waveguide mode having a frequency $f=1.221 \mathrm{GHz}$ and a wave vector equal to $3.468 \mu \mathrm{m}^{-1}$. Figure 4 corresponds to a top view and a threequarter view of the three-dimensional displacement field in the $x y$ plane. It clearly shows that the acoustic displacement is concentrated within and in the close vicinity of the waveguide. The displacement field is very weak in the rest of the phononic crystal since the mode considered lies within the forbidden band of the phononic crystal plate.

\section{B. PZT/air phononic crystal plate on a silicon substrate}

\section{Perfect phononic crystal plate on silicon substrate}

In the previous section, we considered a freestanding phononic crystal plate made of a square array of holes drilled in a PZT5A matrix. The geometrical characteristics of the plate, i.e., the thickness $h$, the lattice parameter $a$, and the radius of the holes $R$ were of the order of micrometers and were chosen in the aim of observing an absolute band gap around a frequency of $1.5 \mathrm{GHz}$. A micrometer thick plate cannot be used effectively in device structures. The physical realization of a practical structure would then require that the thin plate of phononic crystal be deposited onto a thick substrate for support. In consequence, we investigate here the influence of a substrate on the absolute band gap of the

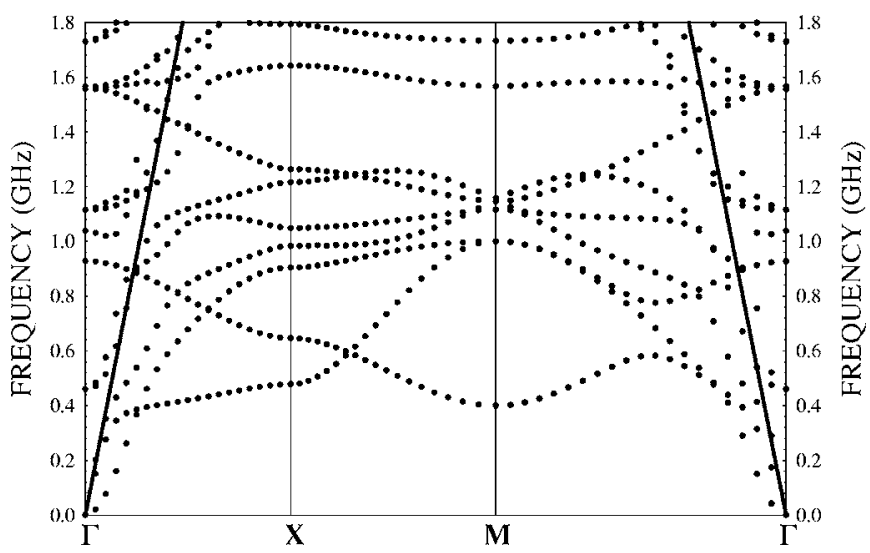

FIG. 5. Elastic band structures for the air/PZT phononic crystal plate of thickness $h=a$ deposited onto a silicon substrate of thickness $d=2 h=2 a$. The thick solid lines represent the dispersion curves of the slower elastic waves propagating in an infinite, homogeneous medium composed of silicon, i.e., the waves of transverse polarization.

phononic crystal plate. We consider a substrate of finite thickness $d$ made of silicon. Figure 5 shows the finite element band structure of the plate deposited on the substrate for $d=2 h=2 a$. The thick solid straight lines represent the dispersion curves of the slower elastic waves propagating in an infinite, homogeneous medium composed of silicon, i.e., the waves of transverse polarization. One first observes that the substrate vibrational modes strongly interact with those of the phononic crystal plate, leading to very different dispersion curves with respect to those of a freestanding plate [see Fig. 2(b)]. Moreover, only modes below the dispersion curves of the transverse waves in silicon are confined inside the phononic crystal plate. With this restriction, the band structure of Fig. 5 appears to exhibit forbidden bands, in particular, along the $X M$ direction of propagation. In the other directions of the Brillouin zone, the plate modes crossover in the vicinity of the $C_{t}$ line of the substrate around $1.5 \mathrm{GHz}$, for example, and appear below the $C_{t}$ line. In Fig. 6 , we present the same band structure but for a thicker substrate, i.e., for $d=5 h=5 a$. In that case, one observes that, in the same range of frequency, some plate modes still appear below the $C_{t}$ line of the substrate but occur very closely to this line. This results in the existence of an absolute forbidden band centered around $f=1.5 \mathrm{GHz}$. Our finite element

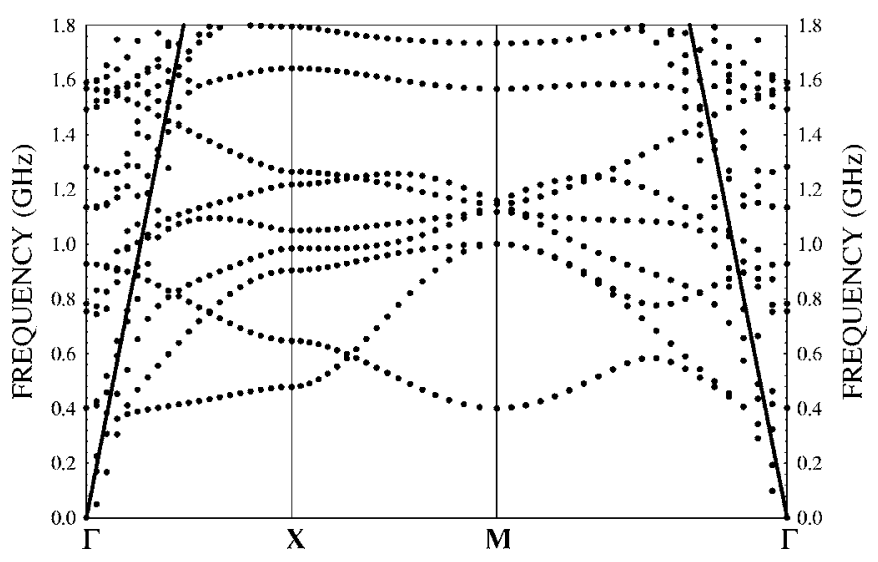

FIG. 6. Same as Fig. 5 but for a substrate thickness $d=5 h=5 a$. 

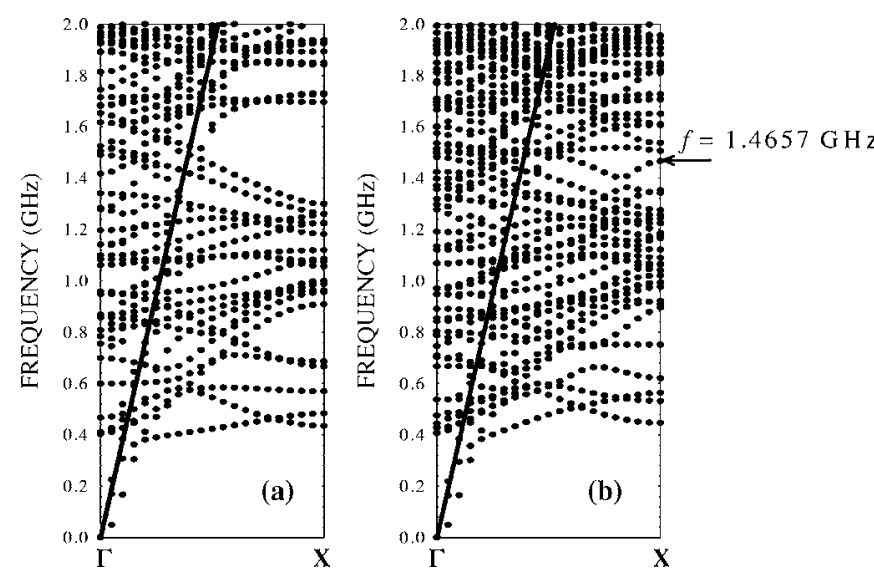

FIG. 7. Band structure along the $\Gamma X$ direction calculated with a supercell containing $1 \times 5$ unit cell, for (a) the perfect phononic crystal plate deposited on the silicon substrate and (b) the supported plate containing a waveguide formed by filling a hole in the third unit cell. The thickness of the substrate is the same as in Fig. 6, i.e., $d=5 h=5 a$. The arrow indicates the location of the guided mode analyzed in Figs. 8(a) and 8(b).

method (FEM) results also show that for much thicker substrate, the dispersion curves below $C_{t}$ remains the same. Our study shows also that the existence of such an absolute forbidden band requires very constraining conditions on the nature of the materials constituting the phononic crystal and the substrate.

\section{Waveguide in the plate deposited on a silicon substrate}

We investigate now the propagation of elastic waves through a rectilinear waveguide created inside the phononic crystal plate deposited on the silicon substrate. The rectilinear waveguide is created as in Sec. III A 2, and the finite element calculations are conducted in the same way. Nevertheless, in that case the computations were done considering a $1 \times 5$ supercell. This allows one to reduce the computation time but does not affect significantly the numerical results. The band structures are calculated along the $\Gamma X$ path of the irreducible Brillouin zone [Fig. 7(b)]. As a matter of comparison, we present also in Fig. 7(a) the band structure of the perfect plate deposited on the substrate computed with a 1 $\times 5$ supercell. The thickness of the plate and of the substrate are $h=a$ and $d=5 h=5 a$, respectively. Figure 7(b) shows that some new modes occur in the forbidden band of Fig. 7(a). To show the confinement of such modes inside the waveguide, we focus our attention on the mode with frequency $f$ $=1.4657 \mathrm{GHz}$ at the $X$ point of the Brillouin zone [indicated by an arrow in Fig. 7(b)]. The modulus of the displacement field associated with this mode is displayed on the map of Fig. 8. This map unequivocally shows the localized nature of this mode indicating that it corresponds to a guided mode of the plate. The three-dimensional representation of the displacement field demonstrates that this guided mode is confined in the plate and does not leak out into the substrate.

\section{CONCLUSIONS}

The purpose of this paper was to investigate the possibility of designing phononic crystal-based devices for tele-
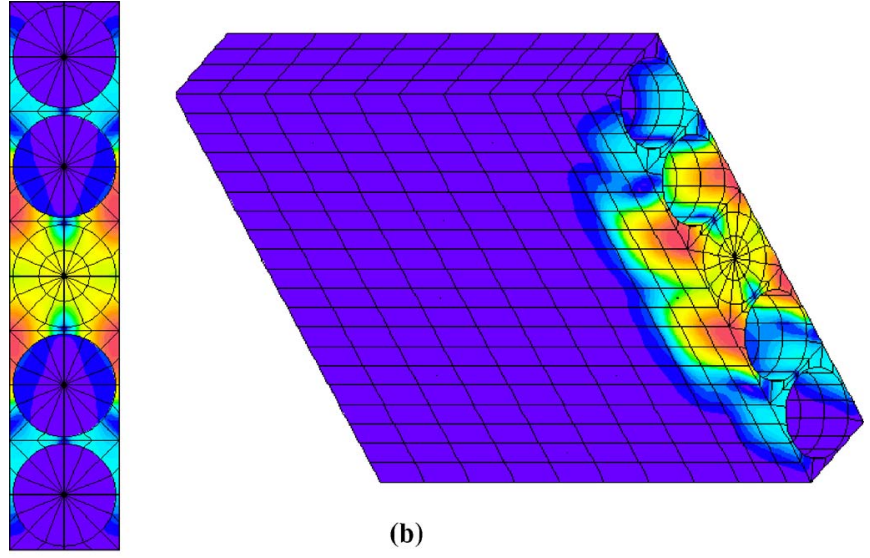

(a)

(b)

FIG. 8. (Color online) Maps of the modulus of the elastic displacement field for the waveguide mode with $f=1.4657 \mathrm{GHz}$ at the $X$ point in Fig. 7(b); (a) top view and (b) three-quarter view. The red color corresponds to the maximum displacement, whereas the blue color corresponds to the minimum.

communication applications using commonly employed materials in microfabrication. We focused our attention on active piezoelectric PZT5A material and silicon. We demonstrated that absolute band gaps for the propagation of plate modes exist in PZT/air hole phononic crystal plates. Incidentally, we also show that plate modes can be guided inside a linear defect created by removing one row of holes. However, practical devices would require that the phononic crystal plate be supported by a substrate. We therefore calculated the band structure of a PZT/air phononic crystal plate on a silicon substrate. Absolute forbidden band in the plate modes forms when the substrate thickness exceeds significantly that of the plate. This gap includes the ubiquitous telecommunication frequency of $1.5 \mathrm{GHz}$. We prove also that a linear defect in such a structure can guide plate mode. The present work provides evidences that phononic crystal properties can be integrated with existing silicon based microdevice technology. This study also suggests that other structural defects such as point defects, cavities, and various channels inserted inside the phononic crystal plate could also lead to the existence of vibrational modes inside the absolute stop bands. These defect modes could then be used to realize functional acoustic devices such as specific filters or demultiplexers.

\section{ACKNOWLEDGMENTS}

This work was supported by Le Fond Européen de Développement Régional (FEDER) and by Le Conseil Régional Nord-Pas de Calais. We acknowledge the assistance of M. Deymier with some of the graphic arts. We also thank P. Mosbah (ISEN, Lille) for his help with the finite element numerical computations.

${ }^{1}$ J. O. Vasseur, B. Djafari-Rouhani, L. Dobrzynski, and P. A. Deymier, J. Phys.: Condens. Matter 9, 7327 (1997).

${ }^{2}$ D. García-Pablos, M. Sigalas, F. R. Montero de Espinosa, M. Torres, M. Kafesaki, and N. García, Phys. Rev. Lett. 84, 4349 (2000).

${ }^{3}$ S. Yang, J. H. Page, Z. Liu, M. L. Cowan, C. T. Chan, and P. Sheng, Phys. Rev. Lett. 93, 024301 (2004).

${ }^{4}$ Y. Pennec, B. Djafari-Rouhani, J. O. Vasseur, A. Khelif, and P. A. Deymier, Phys. Rev. E 69, 046608 (2004). 
${ }^{5}$ Y. Pennec, B. Djafari-Rouhani, J. O. Vasseur, H. Larabi, A. Khelif, A. Choujaa, S. Benchebane, and V. Laude, Appl. Phys. Lett. 87, 261912 (2005).

${ }^{6}$ A. Khelif, A. Choujaa, S. Benchabane, B. Djafari-Rouhani, and V. Laude, Z. Kristallogr. 220, 836 (2005).

${ }^{7}$ T. Miyashita and C. Inoue, Jpn. J. Appl. Phys., Part 1 40, 3488 (2001).

${ }^{8}$ T. Miyashita, Meas. Sci. Technol. 16, R47 (2005).

${ }^{9}$ Y. Tanaka and S. Tamura, Phys. Rev. B 58, 7958 (1998).

${ }^{10}$ Y. Tanaka and S. Tamura, Phys. Rev. B 60, 13294 (1999).

${ }^{11}$ T. T. Wu, Z. G. Huang, and S. Lin, Phys. Rev. B 69, 094301 (2004).

${ }^{12}$ V. Laude, M. Wilm, S. Benchabane, and A. Khelif, Phys. Rev. E 71, 036607 (2005)

${ }^{13}$ T. T. Wu, Z. G. Huang, and S. Y. Liu, Z. Kristallogr. 220, 841 (2005); T. T. Wu, L. C. Wu, and Z. G. Huang, J. Appl. Phys. 97, 094916 (2005).

${ }^{14}$ S. Benchabane, A. Khelif, J.-Y. Rauch, L. Robert, and V. Laude, Phys. Rev. E 73, 065601 (2006).
${ }^{15}$ J. J. Chen, B. Qin, and J. C. Cheng, Chin. Phys. Lett. 22, 1706 (2005).

${ }^{16}$ X. Zhang, T. Jackson, E. Lafond, P. Deymier, and J. O. Vasseur, Appl. Phys. Lett. 88, 041911 (2006).

${ }^{17}$ J. O. Vasseur, P. A. Deymier, B. Djafari-Rouhani, and Y. Pennec, Proceedings of IMECE 2006, ASME International Mechanical Engineering Congress and Exposition, Chicago, Illinois, 5-10, November 2006.

${ }^{18}$ J. C. Hsu and T. T. Wu, Phys. Rev. B 74, 144303 (2006).

${ }^{19}$ A. Khelif, B. Aoubiza, S. Mohammadi, A. Adibi, and V. Laude, Phys. Rev. E 74, 046610 (2006)

${ }^{20}$ The band structures were computed with the help of the ATILA finite element code, ATILA Finite Element Code for Piezoelectric and Magnetostrictive Transducers Modeling, Version 5.2.1, User's Manual, ISEN, Acoustics Laboratory, Lille, France (2002).

${ }^{21} \mathrm{Ph}$. Langlet, A.-C. Hladky-Hennion, and J.-N. Decarpigny, J. Acoust. Soc. Am. 98, 2792 (1995). 